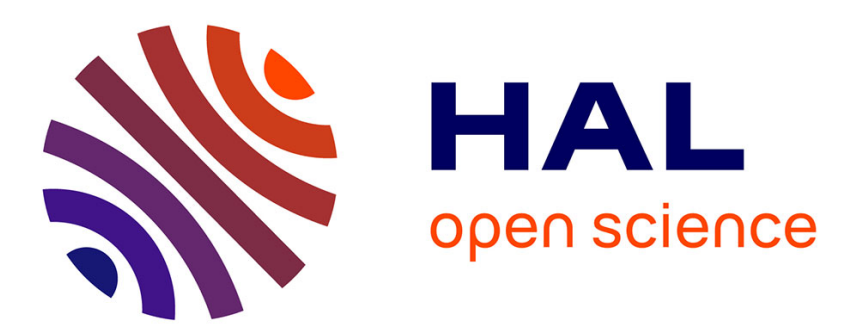

\title{
Stability and traction optimization of a wheel-legged robot
}

Christophe Grand, Faiz Ben Amar, Philippe Bidaud, Frédéric Plumet

\section{To cite this version:}

Christophe Grand, Faiz Ben Amar, Philippe Bidaud, Frédéric Plumet. Stability and traction optimization of a wheel-legged robot. The International Journal of Robotics Research, 2004, 23 (10-11), pp.1041-1058. 10.1177/0278364904047616 . hal-03135896

\section{HAL Id: hal-03135896 https://hal.science/hal-03135896}

Submitted on 29 Mar 2021

HAL is a multi-disciplinary open access archive for the deposit and dissemination of scientific research documents, whether they are published or not. The documents may come from teaching and research institutions in France or abroad, or from public or private research centers.
L'archive ouverte pluridisciplinaire HAL, est destinée au dépôt et à la diffusion de documents scientifiques de niveau recherche, publiés ou non, émanant des établissements d'enseignement et de recherche français ou étrangers, des laboratoires publics ou privés. 


\title{
Stability and traction optimization of a reconfigurable wheel-legged robot
}

\author{
Ch. Grand, F. BenAmar, F. Plumet, Ph. Bidaud \\ Laboratoire de Robotique de Paris (LRP) \\ CNRS FRE2507 - Université de Pierre et Marie Curie, Paris 6 \\ 18, route du Panorama, BP61, 92265 Fontenay-aux-Roses, France \\ \{grand,amar,plumet,bidaud\}@robot.jussieu.fr
}

\section{Revised version}

\begin{abstract}
Actively articulated locomotion systems such as hybrid wheel-legged vehicles are a possible way to enhance the locomotion performance of autonomous mobile robot. In this paper, we address the control of the wheel-legged robot Hylos traveling on irregular sloping terrain. The redundancy of such system is used to optimize both the balance of traction forces and the tipover stability. The general formulation of this optimization problem is presented and a suboptimal but computationally efficient solution is proposed. Then, an algorithm to control the robot posture, based on a velocity model, is described. Finally, this algorithm is validated through simulations and experiments that show the capabilities of such redundantly actuated vehicle to enhance its own safety and autonomy in critical environments.
\end{abstract}

\section{Introduction}

Autonomous exploration missions require mobile robots that can carry out high performance locomotion tasks while insuring the system safety. For applications such as planetary or volcanic exploration or various missions in hazardous areas or construction sites, the locomotion performance in terms of power consumption, autonomy and reliability is of primary importance. Vehicle motion on uneven surfaces involves complex wheel-ground interactions that are related to the geometrical and physical soil properties: roughness, rocks distribution, soil compaction, friction characteristics, etc... 
Therefore, enhancing the locomotion performance in such environment requires the design of innovative locomotion systems and the research of original control schemes.

Current locomotion systems can roughly be divided into wheeled and legged systems. Wheeled robots traveling on natural rough terrain usually use passive internal mobilities. The main research activity in this domain concerns the design of innovative steering (Nomad [20]) and suspension systems. The Rocky rovers [25] and the Shrimp [5], developed respectively at the JPL and EPFL, illustrate the use of passive suspension systems offering high terrain adaptability. These suspensions allow the vehicle to traverse more challenging terrain including ground discontinuities higher than the wheel radius. The main advantage of wheeled locomotion systems is its performance in terms of power consumption, velocity and available payload.

Legged robots are a possible way to increase the field of accessible terrains for autonomous vehicles $[16,22]$. The main activity in this research field concerns the control of complex kinematic structures by considering gait schemes and stability margins. The main relevance of walking machines is their abilities to adapt their posture on uneven terrain and to cross over high terrain discontinuities. And another approach to rough terrain mobility is proposed in [21] with the compliant-legged hexapod Rhex.

To enhance motion capabilities of wheeled robots on irregular and unknown terrains, Wheeled and Actively Articulated Vehicles (WAAV) have been considered. These vehicles are referred as high mobility robots since they possess internal active mobility degrees of freedom, and are illustrated by the WAAV presented in [23] and the Marsokhod [1, 13] robot. They use wheels for propulsion and internal articulation to adapt their configuration. The Hybrid Wheel-Legged Vehicles (HWLV) is a subclass of WAAV that consist of any combination of wheeled and legged mechanisms. The Rollerwalker[9], Workpartner[8], Azimut[18] and Hylos[3] are typical examples of such robots. As the leg's and wheel's degrees of freedom are independently actuated, these systems have the ability to control their posture. In the case of HWLV, the posture is usually defined as the position and orientation of the main body with respect to the ground and the two sideway wheelbases (the distance between each wheel pair in the sagittal plane). As a counterpart, the control of these redundantly actuated systems exhibiting complex interactions with the environment is much more difficult than for conventional wheeled mobile robots.

Control approaches are usually based on modeling and analysis of vehicle motion. Kineto-static analysis of such WAAV has already been addressed by previous authors [23]. Solutions for specific kinematics are presented, and studied in simulation. In [24], a mathematical analysis leads to a model-based control that considers the problem of contact forces distribution in the case of the GOFOR mini-rover (with four internal active mobility degrees). This work however considers only planar vehicle motion and was not experimentally validated. More recently, research on the control of articulated suspension vehicle was considered [12]. The authors proposed a method for stabilitybased articulated suspension control, which is experimentally demonstrated on the SRR robot of JPL. They address tipover stability in the case of SRR robot (with two internal 
active mobility degrees). By also considering the motion of a 3 DOF arm manipulator mounted on the platform, they improve tipover stability.

In this paper, we describe a solution that optimizes both the traction force balance and the tipover margin for the Hylos robot, a high mobility redundantly actuated vehicle developed in the lab (see Figure 1). It is a lightweight mini-robot with 16 actively actuated degrees of freedom (four wheel-legs, each one combining a two degrees of freedom suspension mechanism with a steering and driven wheel).

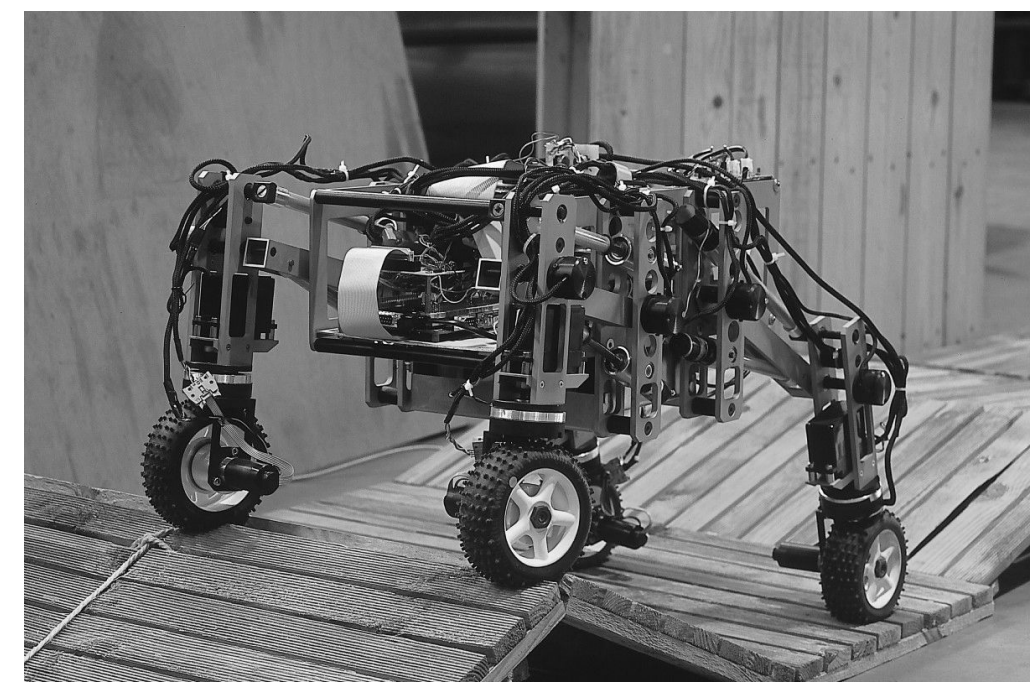

Figure 1: Hylos experimental prototype

The actuated degrees of freedom of this robot are split in two categories: the first one concerns the locomotion itself (traction and steering) and the second one the posture (orientation of the main body and sideway wheelbases). Thus, trajectory and posture control will be treated together as they are coupled but specification of the desired trajectory and optimal posture $\mathbf{p}^{*}$ will be considered separately. The posture control algorithm calculate the joint velocities $\dot{\mathbf{q}}^{*}$ to reach the desired posture $\mathbf{p}^{*}$ and follow the desired trajectory (see Figure 2). The number of posture parameters is related to the general mobility index of the vehicle which depends on the number of internal active mobilities and, thus, on the particular design of the vehicle.

In the next section, we first develop the general kinetostatic model of a wheel-legged vehicle. In Section 3, we define suitable locomotion criteria and address the posture optimization problem, assuming quasi-static motion of the vehicle. However, due to the underlying difficulty of on-line optimal posture computation, a suboptimal but computationally efficient posture for the particular design of the Hylos robot is presented in Section 4 as well as a velocity based posture control algorithm. Finally, performance of the optimization process is analyzed through simulations of a wheel-legged vehicle on sloping terrain with various slope angles. The posture control algorithm is also evaluated through simulations and experiments with the Hylos robot traveling on an irregular and sloping ground. 


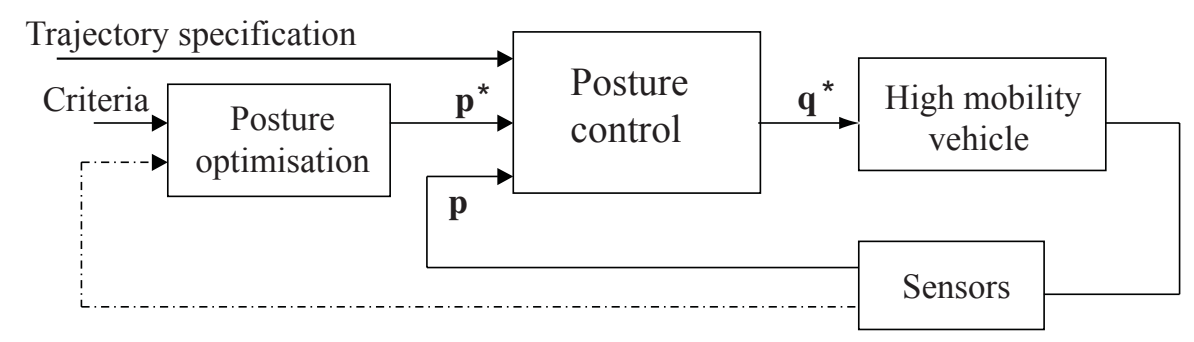

Figure 2: General schematic of the controller

\section{General formulation of kinetostatic model of wheel- legged systems}

This section deals with the development of the kinetostatic model for a general wheeled and articulated system. These models are used in Section 3 for load distribution optimization and in Section 4 for the vehicle posture control on rough terrain.

The considered system consists of a main body (platform S) connected to serial articulated chains, each one terminated by a cylindrical wheel (Figure 3). Define $\mathcal{R}=(\mathrm{G}, \mathbf{x}, \mathbf{y}, \mathbf{z})$ a frame attached to the platform with $\mathrm{G}$ the platform center of gravity (c.o.g). The orientation of the platform frame is given by three angles with respect to the fixed frame $\mathcal{R}_{0}$, which are the conventional yaw $(\theta)-\operatorname{pitch}(\psi)$-roll $(\varphi)$ angles [4].

We assume that all wheels are in contact with the ground. Denote $\mathrm{P}_{\mathrm{i}}$ the $\mathrm{i}^{\mathrm{th}}$ contact point and $\mathbf{n}_{\mathbf{i}}$ the normal vector to the tangent contact plane. The associated contact frame $\mathcal{R}_{\mathrm{i}}=\left(\mathrm{P}_{\mathrm{i}}, \mathbf{t}_{\mathbf{i}}, \mathbf{l}_{\mathbf{i}}, \mathbf{n}_{\mathbf{i}}\right)$ is defined such as $\mathbf{t}_{\mathbf{i}}=\frac{\boldsymbol{\sigma}_{\mathbf{i}} \times \mathbf{n}_{\mathbf{i}}}{\left\|\boldsymbol{\sigma}_{\mathbf{i}} \times \mathbf{n}_{\mathbf{i}}\right\|}\left(\boldsymbol{\sigma}_{\mathbf{i}}\right.$ is the $\mathrm{i}^{\text {th }}$ wheel axis unit vector) and $\mathbf{l}_{\mathbf{i}}=\mathbf{n}_{\mathbf{i}} \times \mathbf{t}_{\mathbf{i}}$.

\section{$2.1 \quad$ Velocity model}

The velocity of each contact point $\mathrm{P}_{\mathrm{i}}$ with respect to the ground can be written as:

$$
\mathbf{v}_{\left(\mathrm{P}_{\mathrm{i}} / \mathcal{R}_{0}\right)}=\mathbf{v}+\boldsymbol{\omega} \times \mathbf{r}_{\mathbf{i}}+\mathbf{v}_{\left(\mathrm{P}_{\mathrm{i}} / \mathcal{R}\right)}
$$

where $(\mathbf{v}, \boldsymbol{\omega})^{\mathrm{t}}=\mathbf{v}_{\mathbf{p}}$ is the components vector expressing the twist of the platform and $\mathbf{r}_{\mathbf{i}}$ the vector connecting the platform frame center $\mathrm{G}$ to contact point $\mathrm{P}_{\mathrm{i}}$.

The pure rolling condition at contact point $\mathrm{P}_{\mathrm{i}}$ can be written as:

$$
\mathbf{v}_{\left(\mathrm{P}_{\mathrm{i}} / \mathcal{R}_{0}\right)}=\mathbf{0}
$$

The projection of this equation on contact frame vectors $\left(\mathbf{t}_{\mathbf{i}}, \mathbf{l}_{\mathbf{i}}, \mathbf{n}_{\mathbf{i}}\right)$ provides different physical meanings: 


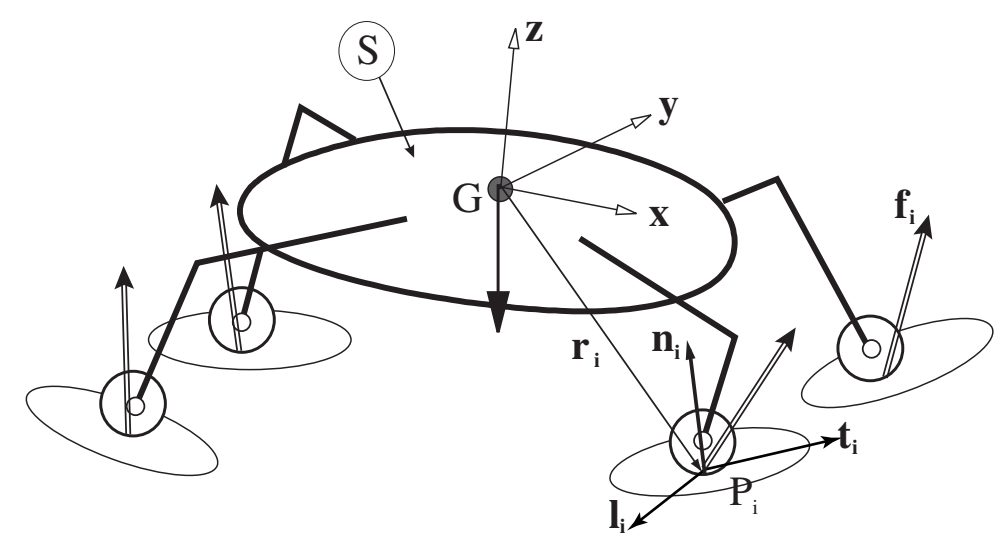

Figure 3: General model of a wheel-legged vehicle

- $\mathbf{t}_{\mathbf{i}}{ }^{\mathrm{t}} \mathbf{v}_{\left(\mathrm{P}_{\mathrm{i}} / \mathcal{R}_{0}\right)}=0$ : expresses the non-slippage condition in the longitudinal direction,

- $\mathbf{l}_{\mathbf{i}}{ }^{\mathrm{t}} \mathbf{v}_{\left(\mathrm{P}_{\mathrm{i}} / \mathcal{R}_{0}\right)}=0$ : expresses the non-slippage condition in the lateral direction,

- $\mathbf{n}_{\mathbf{i}}{ }^{\mathrm{t}} \mathbf{v}_{\left(\mathrm{P}_{\mathrm{i}} / \mathcal{R}_{0}\right)}=0$ : expresses the contact continuity condition.

With the pure rolling condition at contact point $\mathrm{P}_{\mathrm{i}}$, Equation (1) becomes:

$$
-\left(\mathbf{v}+\boldsymbol{\omega} \times \mathbf{r}_{\mathbf{i}}\right)=\mathbf{v}_{\left(\mathrm{P}_{\mathrm{i}} / \mathcal{R}\right)}
$$

and its projection, in a matrix form, along the contact frame vectors yields to:

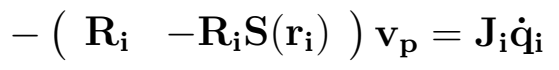

where $\mathbf{R}_{\mathbf{i}}$ is the rotation matrix of the contact frame with respect to platform frame, $\mathbf{J}_{\mathbf{i}}$ is the jacobian matrix of the $i^{\text {th }}$ wheel-leg chain with respect to the platform and expressed in the contact frame, $\dot{\mathbf{q}}_{\mathbf{i}}$ is the joint velocity vector of the wheel-leg chains, and $\mathbf{S}(\mathbf{u})$ is the skew-symmetric matrix of the cross product operator :

$$
\mathbf{S}(\mathbf{u})=\left(\begin{array}{ccc}
0 & -\mathrm{u}_{3} & \mathrm{u}_{2} \\
\mathrm{u}_{3} & 0 & -\mathrm{u}_{1} \\
-\mathrm{u}_{2} & \mathrm{u}_{1} & 0
\end{array}\right)
$$

Equation (4) can be written for each leg as:

$$
\mathbf{L}_{\mathbf{i}} \mathbf{v}_{\mathbf{p}}=\mathbf{J}_{\mathbf{i}} \dot{\mathbf{q}}_{\mathbf{i}}
$$

where $\mathbf{L}_{\mathbf{i}}$ is called the locomotion matrix of the $i^{\text {th }}$ wheel-leg chain.

Finally, for all wheel-legs, we obtain:

$$
\left(\begin{array}{c}
\mathbf{L}_{1} \\
\mathbf{L}_{2} \\
\ldots \\
\mathbf{L}_{\mathbf{n}}
\end{array}\right) \mathbf{v}_{\mathbf{p}}=\left(\begin{array}{cccc}
\mathbf{J}_{1} & 0 & 0 & 0 \\
0 & \mathbf{J}_{2} & 0 & 0 \\
0 & 0 & \ldots & 0 \\
0 & 0 & 0 & \mathbf{J}_{\mathbf{n}}
\end{array}\right)\left(\begin{array}{c}
\dot{\mathbf{q}}_{1} \\
\dot{\mathbf{q}}_{2} \\
\ldots \\
\dot{\mathbf{q}}_{\mathbf{n}}
\end{array}\right)
$$


or

$$
\mathbf{L} \mathbf{v}_{\mathbf{p}}=\mathbf{J} \dot{\mathbf{q}}
$$

\section{$2.2 \quad$ Static model}

We denote $\mathbf{f}_{\mathbf{i}}=\left(f_{t_{i}}, f_{l_{i}}, f_{n_{i}}\right)^{\mathrm{t}}$ the contact force at point $\mathrm{P}_{\mathrm{i}}$ expressed in the contact frame $\mathcal{R}_{\mathrm{i}}$. The components of this contact force are called respectively the traction (or braking) force, lateral force and normal force (or load). Equations of static equilibrium are obtained using the principle of virtual work. This gives, on the one hand, the equilibrium equation of the whole system:

$$
\mathbf{L}^{\mathrm{t}} \mathbf{f}=\mathbf{w}_{\mathbf{t}}
$$

and, on the other hand, the contact force to joint's torques $\boldsymbol{\tau}$ mapping:

$$
\mathbf{J}^{\mathrm{t}} \mathbf{f}=\boldsymbol{\tau}+\mathbf{w}_{\mathbf{s}}
$$

where $\mathbf{f}=\left(\mathbf{f}_{\mathbf{1}}{ }^{\mathrm{t}}, \mathbf{f}_{\mathbf{2}}{ }^{\mathrm{t}}, . ., \mathbf{f}_{\mathbf{n}}{ }^{\mathrm{t}}\right)^{\mathrm{t}}$ is the collection of all contact forces.

In these equations, $\mathbf{w}_{\mathbf{t}}$ is the components vector of the total wrench (expressed in the platform frame) applied to the system and $\mathbf{w}_{\mathbf{s}}$ is the generalized force vector mainly due to the weight of the different sub-parts of the system.

\section{Posture optimization of wheel-legged vehicle}

The locomotion performance of wheel-legged vehicles is directly related to the contact forces at each wheel-ground contact point. The allowable range of these contact forces depends on the vehicle posture. Thus, posture control in articulated ground vehicle is a possible way to enhance the locomotion performance. Controlling the posture can also be used to control the center of gravity position (i.e. the distribution of contact forces) and thus the tipover stability margin.

Estimation of contact forces is based on a quasi-static analysis of the vehicle configuration. Consider the high mobility locomotion system shown in Figure 3: it is a generic model that represents both legged, wheeled and hybrid vehicle. The generic kinematic model developed in Section 2 is used to find the optimal posture for this wheel-legged vehicle. The resolution of forces distribution in closed-chain mechanisms is an underspecified problem [14]. With the assumption that the wheel-ground contact angles can be estimated [11, 2], the quasi-static equilibrium equation (Eq. (8)) gives the force balance for a given configuration, using the pseudo-inverse of $\mathbf{L}^{\mathrm{t}}$. 


\subsection{Locomotion performance criteria}

To find the posture vector $\mathbf{p}^{*}$ that optimizes the vehicle locomotion performance, it is necessary to select suitable evaluation criteria. In planetary exploration missions, fundamental properties that should be enhanced are the vehicle reliability and its capacity to travel in difficult environment. Thus, in this paper, the mechanical stability margin (the system tipover limit) and the traction efficiency are considered to be the most suitable performance criteria.

Stability criterion: The control of robotic systems under stability margin conditions was mainly addressed in the field of legged locomotion. Research on stability control of walking machines was first considered in 1968 by McGhee and Frank [15]. A first static stability criterion was developed to evaluate the stability of an ideal machine walking at constant speed on flat even terrain. It simply considers that the vehicle is stable if the projection of the center of gravity lies inside the support polygon. Different mechanical stability margins were defined during past research on walking machines. They can be roughly split in three main categories [10]:

- the "Stability margin" [15] that evaluates the distance between the projection of the center of gravity and the support polygon,

- the "Gradient stability margin"[19] that evaluates the inclination angle of the machine at which it starts tumbling,

- the "Energy stability margin" $[17,6]$ that evaluates the difference between its maximum potential energy and its initial one.

The control method presented in this paper considers the vehicle motion on irregular terrain without discontinuities. Thus, the tipover stability margin is mainly constrained by terrain geometry. So, a "Gradient stability margin" as the one proposed by Papadopoulos [19] is well adapted to our operational condition, as it integrates both the distance of the projected c.o.g. to the support polygon and its vertical position relatively to the average plane defined by the contact points $\mathrm{P}_{\mathrm{i}}$. Furthermore, this method is computationally efficient. It can be summarized as follow: the line joining two consecutive terrain-contact points $\mathrm{P}_{\mathrm{i}}$ define a tip-over axis. The unit vector $\mathbf{h}_{\mathbf{i}}$ of the axis joining the vehicle c.o.g. $G$ to the center of each tipover axis is computed. Then, angle $\theta_{i}$ between each $\mathbf{h}_{\mathbf{i}}$ and the total external force vector applied to the vehicle gives the stability angle over the corresponding tipover axis. The overall vehicle stability margin is defined as the minimum of all stability angles:

$$
m_{s}=\min \left\{\theta_{i}, i=1 . . n\right\}
$$

When $m_{s}<0$, a tipover instability occurs.

Traction criterion: Consider the contact force $\mathbf{f}_{\mathbf{i}}=\left(f_{t_{i}}, f_{l_{i}}, f_{n_{i}}\right)^{\mathrm{t}}$ as defined in Section 2 and expressed in the local contact frame $\mathcal{R}_{\mathrm{i}}$. Denote $\rho_{i}$ the ratio between 
tangential and normal forces at each wheel-ground contact:

$$
\rho_{i}=\frac{\sqrt{f_{l_{i}}^{2}+f_{t_{i}}^{2}}}{f_{n_{i}}}
$$

Traction efficiency is related to the slip that occurs at each wheel-ground contact. Reducing the potential for large-scale slippage is equivalent to minimize the maximum of each ratio $\rho_{i}$. The limit of controllability is reached when $\rho_{i} \geq f_{s}$, where $f_{s}$ is the wheel-terrain friction coefficient.

\subsection{Formulation of the optimization problem}

For a given set of posture parameters $\mathbf{p}$ (which depends on the particular design of the vehicle), the aim of the optimization process is to find the optimal posture vector $\mathbf{p}^{*}$ which minimizes an objective function $\Phi$.

The objective function $\Phi(\mathbf{p})$ can be expressed as a function of the locomotion performance criteria:

$$
\Phi(\mathbf{p})=\sum_{i=1}^{n}\left(\frac{K_{s}}{\theta_{i}^{2}}+K_{\rho} \rho_{i}^{2}\right)
$$

where $\rho_{i}$ is a function of $\mathbf{f}_{\mathbf{i}}$ and $\theta_{i}$ a function of $\mathbf{r}_{\mathbf{i}}$ (vector connecting the c.o.g to each contact point), and $K_{s}, K_{\rho}$ are constant positive weighting factors. Minimizing this function leads to maximize all the $\theta_{i}$ (i.e. the margin stability)and minimize all the $\rho_{i}$ (i.e. the total vehicle slippage).

The vectors $\mathbf{r}_{\mathbf{i}}$ are computed as a function of $\mathbf{p}$ and the static equilibrium equation (Eq. (8)) is solved using the pseudo-inverse matrix to determine $\mathbf{f}$ :

$$
\mathbf{f}=\left(\mathbf{L}^{\mathrm{t}}\right)^{+} \mathbf{w}_{\mathbf{t}}
$$

A standard conjugate gradient method could then be used to search for the optimal posture.

However, in order to evaluate the objective function, we need information about the local terrain map to define the contact points $P_{i}$ and the associated normal vectors $\mathbf{n}_{\mathbf{i}}$. As the terrain map is generally unknown, $\mathrm{P}_{\mathrm{i}}$ and $\mathbf{n}_{\mathbf{i}}$ have to be evaluated on line. Thus, the main drawback of this method lies on its practical issues: measurement of the contact normals and computational cost of the on-line optimization process. Thus, for the practical implementation of the posture control algorithm on the Hylos robot, a suboptimal solution is proposed in the next section. 


\section{Suboptimal posture definition and control of $\mathrm{Hy}-$ los}

In this section, we describe the method used for the posture control of Hylos robot. We use a proportional feedback control based on the inverse velocity model. We first define, through a kinematic analysis, the parameters of the posture vector $\mathbf{p}$ and then we give the general inverse model used for both path tracking and posture control. Finally, we present the posture control algorithm applied to the Hylos robot as well as the definition of a suboptimal posture for motion on slopes.

\subsection{Hylos posture parameters definition}

The number of posture parameters is related to the mobility of the vehicle which depends on the particular design of the Hylos robot. The mobility $m$ is computed using the Kutzbach form of Gruebler's equation:

$$
m=\sum_{i=1}^{j} f_{i}-6(j-b+1)
$$

where $b$ is the number of bodies, $j$ is the number of joints and $f_{i}$ is the number of freedom for each joint.

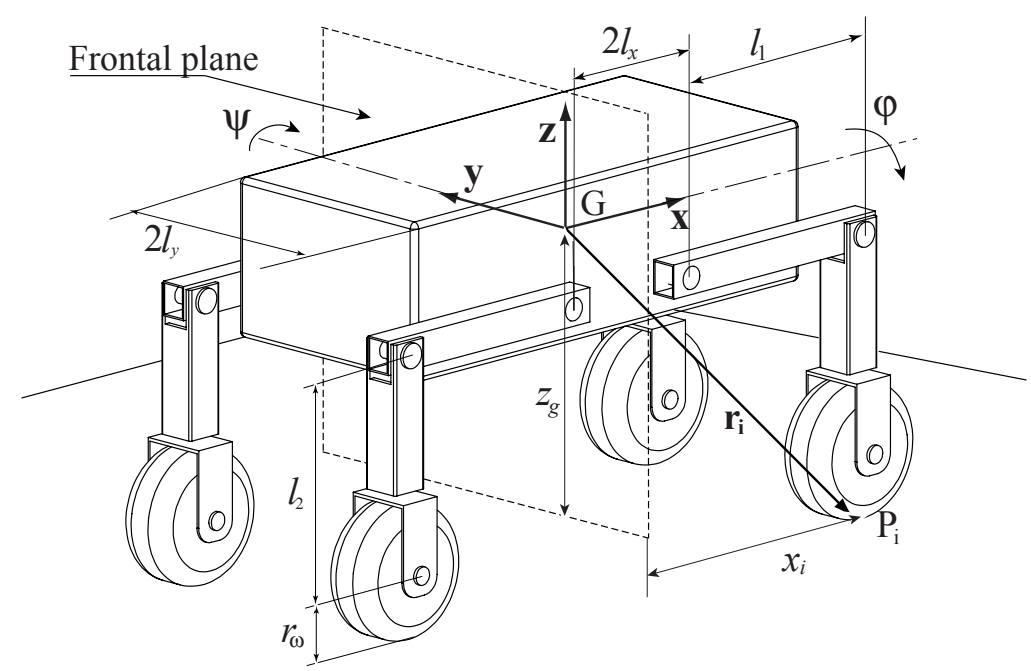

Figure 4: Hylos kinematic model

The Hylos robot, presented in Figure 4, has a mobility $m=10$ with 16 actuated joints: $b=18$ ( 4 bodies for each leg, the platform and the ground), $j=20$ (4 joints on each leg and 4 wheel-ground contacts) and $\sum f_{i}=28$ (4 rotational joints for each leg and 3 dof joints at each wheel-ground contact with ideal rolling constraint). 
In the operational space, these mobilities are the 6 platform parameters $\mathbf{v}_{\mathbf{p}}$ and the 4 wheelbase velocities $\dot{x}_{i}$ of each contact. These parameters can be split into one part $\left(v_{x}, v_{y}, \omega_{z}\right)$ dealing with the path tracking and the other $\left(\omega_{x}, \omega_{y}, v_{z}, \dot{x}_{1}, \dot{x}_{2}, \dot{x}_{3}, \dot{x}_{4}\right)$ with the posture control. Then, the corresponding geometrical posture parameters are:

$$
\mathbf{p}=\left(\varphi, \psi, z_{g}, x_{1}, x_{2}, x_{3}, x_{4}\right)^{\mathrm{t}}
$$

where :

- $\varphi$ is the roll angle,

- $\psi$ is the pitch angle,

- $z_{g}$ is the height of platform center of gravity relative to the ground and is defined as the average of contact heights $z_{i}: z_{g}=\frac{\sum_{i} z_{i}}{4}$

- $x_{i}$ is the wheelbase of each wheel.

\subsection{Hylos inverse velocity model}

Let us consider the wheel-leg chain kinematics given in Figure 5: $\alpha_{i}, \beta_{i}$ are the leg's joint angles, $\gamma_{i}$ the steering angle and $\omega_{i}$ the wheel rate. Equation (3) expressed for the Hylos robot becomes:

$$
-\left(\mathbf{v}+\boldsymbol{\omega} \times \mathbf{r}_{\mathbf{i}}\right)=\dot{\alpha}_{i} \mathbf{y} \times \mathbf{a}_{\mathbf{i}}+\dot{\beta}_{i} \mathbf{y} \times \mathbf{b}_{\mathbf{i}}+\dot{\gamma}_{i} \boldsymbol{\mu}_{\mathbf{i}} \times \mathbf{c}_{\mathbf{i}}+\omega_{i} \boldsymbol{\sigma}_{\mathbf{i}} \times \mathbf{d}_{\mathbf{i}}
$$

where $\boldsymbol{\mu}_{\mathbf{i}}, \boldsymbol{\sigma}_{\mathbf{i}}$ are the unit vectors of the steering and wheel axis, and $\mathbf{a}_{\mathbf{i}}, \mathbf{b}_{\mathbf{i}}, \mathbf{c}_{\mathbf{i}}, \mathbf{d}_{\mathbf{i}}$ the vectors connecting the joint axis to the contact point.

Due to the steering joint kinematics, the steering axis is almost perpendicular to the contact surface. Then the cross product $\boldsymbol{\mu}_{\mathbf{i}} \times \mathbf{c}_{\mathbf{i}}=\boldsymbol{\mu}_{\mathbf{i}} \times-r_{w} \mathbf{n}_{\mathbf{i}}$ is almost null which means that the steering rate $\dot{\gamma}_{i}$ has no noticeable effect on the instantaneous motion of the platform. The normal vector is assumed to be in the wheel plane, then $\boldsymbol{\sigma}_{\mathbf{i}}=\mathbf{l}_{\mathbf{i}}$ and $\omega_{i} \boldsymbol{\sigma}_{\mathbf{i}} \times \mathbf{d}_{\mathbf{i}}=\omega_{i} \mathbf{l}_{\mathbf{i}} \times\left(-r_{w} \mathbf{n}_{\mathbf{i}}\right)=-r_{w} \omega_{i} \mathbf{t}_{\mathbf{i}}$. Assuming these conditions, Equation (3) becomes:

$$
-\left(\mathbf{v}+\boldsymbol{\omega} \times \mathbf{r}_{\mathbf{i}}\right)=\dot{\alpha}_{i} \mathbf{y} \times \mathbf{a}_{\mathbf{i}}+\dot{\beta}_{i} \mathbf{y} \times \mathbf{b}_{\mathbf{i}}-r_{w} \omega_{i} \mathbf{t}_{\mathbf{i}}
$$

As in Section 2, this equation, projected in the $\mathrm{i}^{\text {th }}$ contact frame, gives the following matrix form:

$$
\mathbf{L}_{\mathbf{i}} \mathbf{v}_{\mathbf{p}}=\mathbf{J}_{\mathbf{i}} \dot{\mathbf{q}}_{\mathbf{i}}
$$

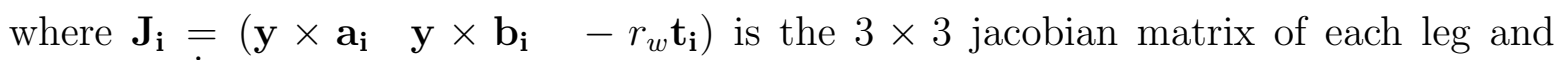
$\dot{\mathbf{q}}_{\mathbf{i}}=\left(\dot{\alpha}_{i}, \dot{\beta}_{i}, \omega_{i}\right)^{\mathrm{t}}$. 


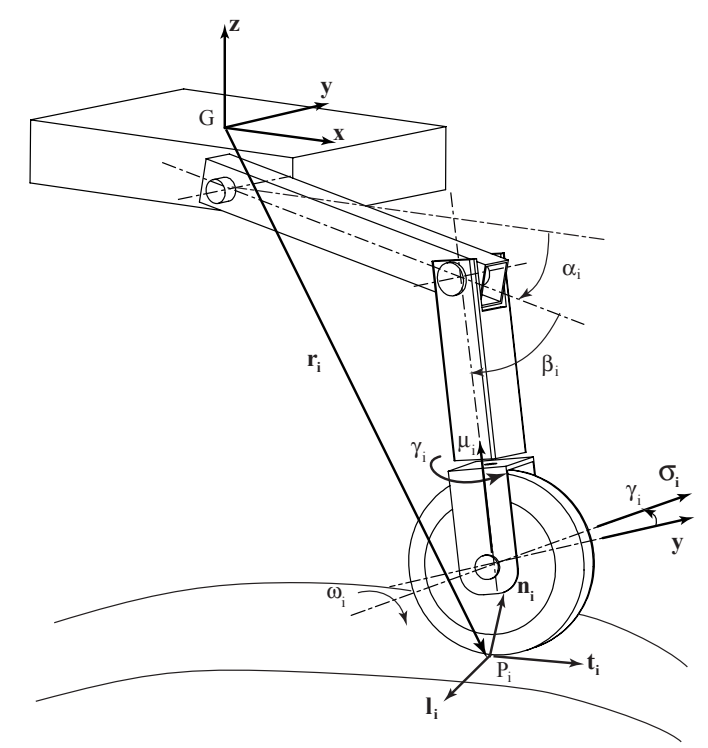

Figure 5: Parameters of a wheel-leg

This equation expresses the contact condition and non-slippage condition along the two tangential directions (see Section 2). The second row scalar equation in Equation (18) expresses the inherent non-holonomic constraint of the wheeled system:

$$
\boldsymbol{\sigma}_{\mathbf{i}}^{\mathrm{t}}\left(\mathbf{v}+\boldsymbol{\omega} \times \mathbf{r}_{\mathbf{i}}+\dot{\alpha}_{i} \mathbf{y} \times \mathbf{a}_{\mathbf{i}}+\dot{\beta}_{i} \mathbf{y} \times \mathbf{b}_{\mathbf{i}}\right)=0
$$

This constraint can be satisfied if we can find the steering angle $\gamma_{i}$ compatible with the system motion. By computing the steering angle $\gamma_{i}$ compatible with the system motion, we reduce the number of velocity parameters to 12. Equation (18), expressed for each leg, gives only 8 scalar equations. As mentioned in the previous section, the wheelbase parameter change $\dot{x}_{i}$ is introduced in order to complete the operational parameter vector. Then, the model of the $i^{\text {th }}$ wheel-legged motion can be written as:

$$
\left(\begin{array}{cc}
\mathrm{BL}_{\mathrm{i}} & \mathbf{0} \\
\mathbf{0} & 1
\end{array}\right)\left(\begin{array}{c}
\mathrm{v}_{\mathbf{p}} \\
\dot{x}_{i}
\end{array}\right)=\left(\begin{array}{c}
\mathrm{BJ}_{\mathrm{i}} \\
\mathrm{j}_{\mathrm{i}}
\end{array}\right) \dot{\mathrm{q}}_{\mathrm{i}}
$$

where $\mathbf{j}_{\mathbf{i}}=\left(-l_{1} \sin \left(\alpha_{i}\right)-l_{2} \sin \left(\alpha_{i}+\beta_{i}\right),-l_{2} \sin \left(\alpha_{i}+\beta_{i}\right), 0\right)$ is a reduced jacobian matrix of the leg expressed in the platform frame (see details in the Appendix) and

$$
\mathbf{B}=\left(\begin{array}{lll}
1 & 0 & 0 \\
0 & 0 & 1
\end{array}\right)
$$

is the reduction matrix used to eliminate the steering angle $\gamma_{i}$.

We then obtain:

$$
\mathbf{L}_{\mathbf{i}}^{*}\left(\begin{array}{c}
\mathbf{v}_{\mathbf{p}} \\
\dot{x}_{i}
\end{array}\right)=\mathbf{J}_{\mathbf{i}}^{*} \dot{\mathbf{q}}_{\mathbf{i}}
$$


$\mathbf{J}_{\mathbf{i}}{ }^{*}$ is a $3 \times 3$ square matrix and can be inverted to give finally the wheel-leg motion by:

$$
\dot{\mathbf{q}}_{\mathbf{i}}=\left(\mathbf{J}_{\mathbf{i}}^{*}\right)^{-1} \mathbf{L}_{\mathbf{i}}^{*}\left(\begin{array}{c}
\mathbf{v}_{\mathbf{p}} \\
\dot{x}_{i}
\end{array}\right)
$$

The projection of the non-holonomic constraint of Equation (19) in the vehicle frame $\mathcal{R}$, gives the steering angle compatible with the vehicle motion (see details of this calculation in the Appendix):

$$
\gamma_{i}=\arctan \left(\frac{v_{i_{y}}^{\prime}}{v_{i_{x}}^{\prime} \sin \left(\alpha_{i}+\beta_{i}\right)-v_{i_{z}}^{\prime} \cos \left(\alpha_{i}+\beta_{i}\right)}\right)
$$

where $\mathbf{v}_{\mathbf{i}}^{\prime}=\left(v_{i_{x}}^{\prime}, v_{i_{y}}^{\prime}, v_{i_{z}}^{\prime}\right)$ corresponds to: $\left(\mathbf{v}+\boldsymbol{\omega} \times \mathbf{r}_{\mathbf{i}}+\dot{\alpha}_{i} \mathbf{y} \times \mathbf{a}_{\mathbf{i}}+\dot{\beta}_{i} \mathbf{y} \times \mathbf{b}_{\mathbf{i}}\right)$.

\subsection{Posture control algorithm}

For a given optimal posture $\mathbf{p}^{*}$ and a desired trajectory, the goal of posture control is to compute the internal joint velocities $\dot{\mathbf{q}}_{\mathbf{i}}$ applied to each motor to maintain the optimal posture during the motion.

Let us introduce $\dot{\mathbf{p}}=\left(\dot{\varphi}, \dot{\psi}, \dot{z}_{g}, \dot{x}_{1}, \dot{x}_{2}, \dot{x}_{3}, \dot{x}_{4}\right)^{\mathrm{t}}$ as the time derivative of posture parameters. Then, the posture control is achieved by using a state feedback linearizing control law:

$$
\dot{\mathbf{p}}=\mathrm{K}_{\mathrm{p}} \Delta \mathrm{p}
$$

where $\Delta \mathbf{p}=\mathbf{p}^{*}-\mathbf{p}$ is the posture error and $\mathbf{K}_{\mathbf{p}}$ is a $7 \times 7$ diagonal positive gain matrix. The three other velocity parameters $\mathbf{v}_{\mathbf{t}}=\left(v_{x}, v_{y}, \dot{\theta}\right)^{\mathrm{t}}$ are used in the trajectory tracking control loop, which is not detailed in this paper.

The term $\dot{z}_{g}$ is a function of $\mathbf{v}_{\mathbf{p}}$ (calculation details of this equation are given in the Appendix):

$$
\dot{z}_{g}=-\mathbf{v}^{\mathrm{t}} \mathbf{z}+\frac{1}{4} \sum_{i} \mathbf{r}_{\mathbf{i}}^{\mathrm{t}}(\mathbf{S}(\boldsymbol{\omega}) \mathbf{z})
$$

Since we have $\sum_{i} x_{i}=0$ and $\sum_{i} y_{i}=0$ for the suboptimal posture defined in Section 4.4, Equation (25) can be approximated as:

$$
v_{z}=-\dot{z}_{g}+\omega_{y} \frac{\sum_{i} x_{i}}{4}-\omega_{x} \frac{\sum_{i} y_{i}}{4} \approx-\dot{z}_{g}
$$

The platform angular velocities $\boldsymbol{\omega}$ are coupled functions of $(\dot{\varphi}, \dot{\psi}, \dot{\theta})^{\mathrm{t}}$. We introduce the coupling matrix $\mathbf{D}$ such as $\mathbf{v}_{\mathbf{p}}=\mathbf{D}\left(v_{x}, v_{y}, v_{z}, \dot{\varphi}, \dot{\psi}, \dot{\theta}\right)^{\mathrm{t}}$ (see detail in the Appendix). This leads to the following matrix form for each leg:

$$
\begin{aligned}
& \mathbf{v}_{\mathbf{p}}=\mathbf{D}\left(\mathbf{C}_{\mathbf{p}} \dot{\mathbf{p}}+\mathbf{C}_{\mathbf{t}} \mathbf{v}_{\mathbf{t}}\right) \\
& \dot{x}_{i}=\mathbf{C}_{\mathbf{x}_{\mathbf{i}}} \dot{\mathbf{p}}
\end{aligned}
$$


where $\mathbf{C}_{\mathbf{p}}, \mathbf{C}_{\mathbf{t}}$ and $\mathbf{C}_{\mathbf{x}_{\mathbf{i}}}$ are the corresponding component selection matrices.

The joint velocities are computed from this operational velocity vector by considering the inverse velocity model described in the previous paragraph. This model is based on knowledge of the contact normal vectors $\mathbf{n}_{\mathbf{i}}$. Equation (17) shows that $\mathbf{t}_{\mathbf{i}}$ can be estimated from the measure of the absolute platform velocity $(\mathbf{v}, \boldsymbol{\omega})$ and the leg's joint velocities $\left(\dot{\alpha}_{i}, \dot{\beta}_{i}\right)$. However, this estimation is theoretically independent from the wheel's rotation rate $\omega_{i}$. For experimental tests, we use a simplified inverse velocity model which is based on a contact normal vector computed from the average plane of contact points $\mathrm{P}_{\mathrm{i}}\left(x_{i}, y_{i}, z_{i}\right)$.

Finally, the joint velocities are computed using Equation (22).

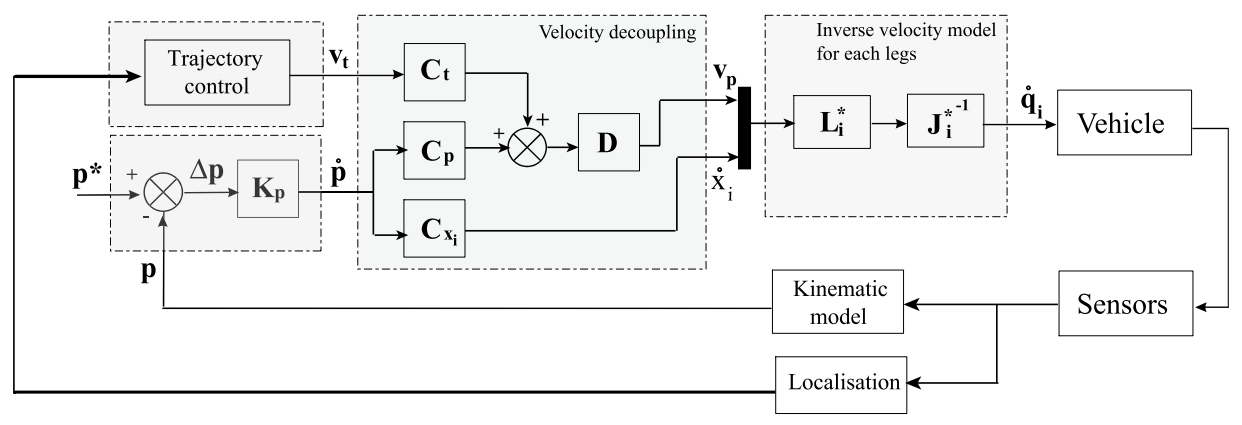

Figure 6: Posture control scheme

\subsection{Suboptimal posture on slope}

Due to the underlying difficulties of the on line optimization scheme presented at the end of Section 3, we here define a suboptimal posture for the motion of Hylos on sloping terrain.

From a static force distribution analysis, we can see that the stability margin and balance of normal contact forces are optimal when the vertical component of the contact forces are equally distributed. It is well known that vertical contact forces balance can be reached by minimizing the projected distance, on horizontal plane, between the vehicle c.o.g and the geometric center of wheel-ground contacts. Moreover, this criterion also optimizes the traction force.

Since the gauge is constant for the particular design of Hylos, it is clear that the sideways force balance, in the front view, is obtained when the roll angle is zero $\varphi=0$. The second constraint concerns a force balance in the sagittal plane between front and rear wheels. For a specified positive nominal wheelbase $x_{n}$, the forces balancing criterion is verified if:

$$
x_{i}=\frac{\overline{x_{n}}}{\cos (\psi)}-z_{g} \tan (\psi)
$$


where $\overline{x_{n}}$ is the algebraic value of $x_{n}$ which is positive for the front wheels and negative for the rear ones.

The vehicle ground clearance $z_{g}$, the pitch angle $\psi$, and the nominal wheelbase $x_{n}$ are specified by the supervision control considering kinematic constraints on obstacle clearance and a constraint involving embedded scientific instruments. For example, it could be necessary to keep the platform horizontal for vision-based measurements.

\section{Results}

In this section, we first present results showing an enhancement of the locomotion performance in terms of stability margin and traction, with the suboptimal posture for motion on sloping ground. We choose a constant ground clearance $z_{g}=-\left(l_{2}+r_{w}\right)$ and pitch angle $\psi=0$. The 5 other parameters of the posture vector are computed using the method presented in the previous section with a nominal wheelbase $x_{n}=l_{x}+l_{1}\left(l_{1}\right.$ and $l_{2}$ are the length of the leg links, $l_{x}$ is the half-length of the platform).

Next, simulation results show the ability of the posture control algorithm to ensure the system stability on highly challenging terrains. Finally, experimental results for the posture control of Hylos moving on irregular terrain (consisting of a succession of slopes with different inclination angles) show the feasibility of this method.

Furthermore, in order to illustrate the enhancement of Hylos locomotion performance, we purpose a comparison between the suboptimal posture configuration and the fixed-leg configuration. This configuration corresponds to a system without internal mobility degrees; i.e. with legs in a fixed position defined by: $\alpha_{i}=$ cte $=0$ and $\beta_{i}=$ cte $=-\pi / 2$. In the rest of this paper, the fixed-leg configuration will be referred to as the nominal posture.

\subsection{Suboptimal posture on a sloping ground}

We denote $\eta_{s}$ the maximum slope angle with respect to the horizontal plane and $\theta_{s}$ the heading angle of the robot $\mathbf{x}$ axis with respect to the line of maximum slope (see Figure 7). Then, the corresponding pitch and roll angles of the robot in the rigid nominal posture are:

$$
\begin{aligned}
& \varphi=\arcsin \left(\sin \left(\theta_{s}\right) \sin \left(\eta_{s}\right)\right) \\
& \psi=\arcsin \left(\cos \left(\theta_{s}\right) \sin \left(\eta_{s}\right)\right)
\end{aligned}
$$

For each pair of angles $\left(\eta_{s}, \theta_{s}\right)$, the static model is solved for the suboptimal posture defined above and for the rigid nominal posture. Then, we compute the stability limit $\left(m_{s}=0\right)$ and the controllability limit $\left(\rho_{\max }=f_{s}\right)$, which is the limit when slipping occurs. 


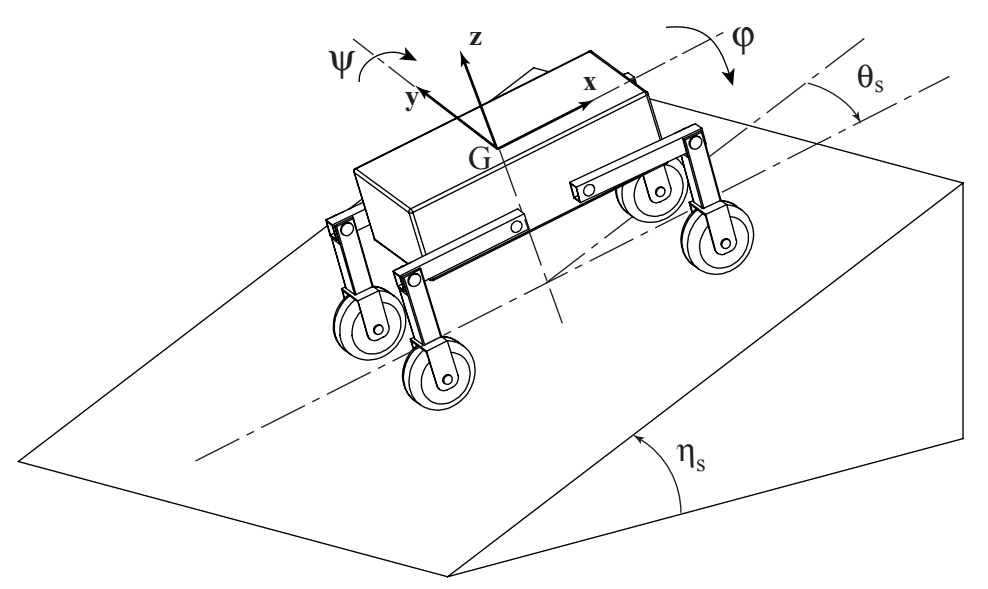

Figure 7: Slope angles definition

The stability and controllability limits are represented on a polar coordinate graph in Figure 8 with $\eta_{s}$ for the radius and $\theta_{s}$ for the angle. Obviously, the stability and controllability domains are much larger for the suboptimal posture case. For the nominal posture, the stability and controllability are smaller for $\theta_{s}=\pi / 2$ as the gauge (vehicle width) is smaller than the wheelbase (vehicle length). When the suboptimal posture is considered, the stability limit is theoretically very important but is in practice mainly constrained by the operational workspace limit of each leg $\left(\left|\alpha_{i}\right|<50^{\circ},\left|\beta_{i}\right|<50^{\circ}\right)$. As the roll angle is constrained to zero, we obtain an isotropic behavior of the controllability limit curve.

\subsection{Simulation results on posture control}

In order to evaluate Hylos locomotion performance, we have developed a simulator [7] that takes into account the dynamics of wheel-legged robot and the wheel ground interactions. The posture control algorithm presented in Section 4 has been implemented and evaluated for the Hylos robot traveling on irregular terrain (see Figure 9).

These simulations have the same initial and boundary conditions in terms of soil properties and robot state. We specify a constant straightforward trajectory with a velocity of $30 \mathrm{~cm} / \mathrm{sec}$. The simulations were performed with suboptimal posture control and without posture control (constant nominal posture defined by $\alpha_{i}=0, \beta_{i}=-\pi / 2$ ). The terrain used for these simulations is roughly irregular with two different surface profiles in each sideways sagittal plane. It is a challenging terrain since without posture control the system exhibits tipover instability.

The pitch and roll angles of the robot with posture control are plotted in Figure 10. This plot shows the performance of posture control for the selected feedback gain. Roll angles, which has the most important impact on the tipover margin, is well controlled, as the maximum error is less than $1^{\circ}$. 


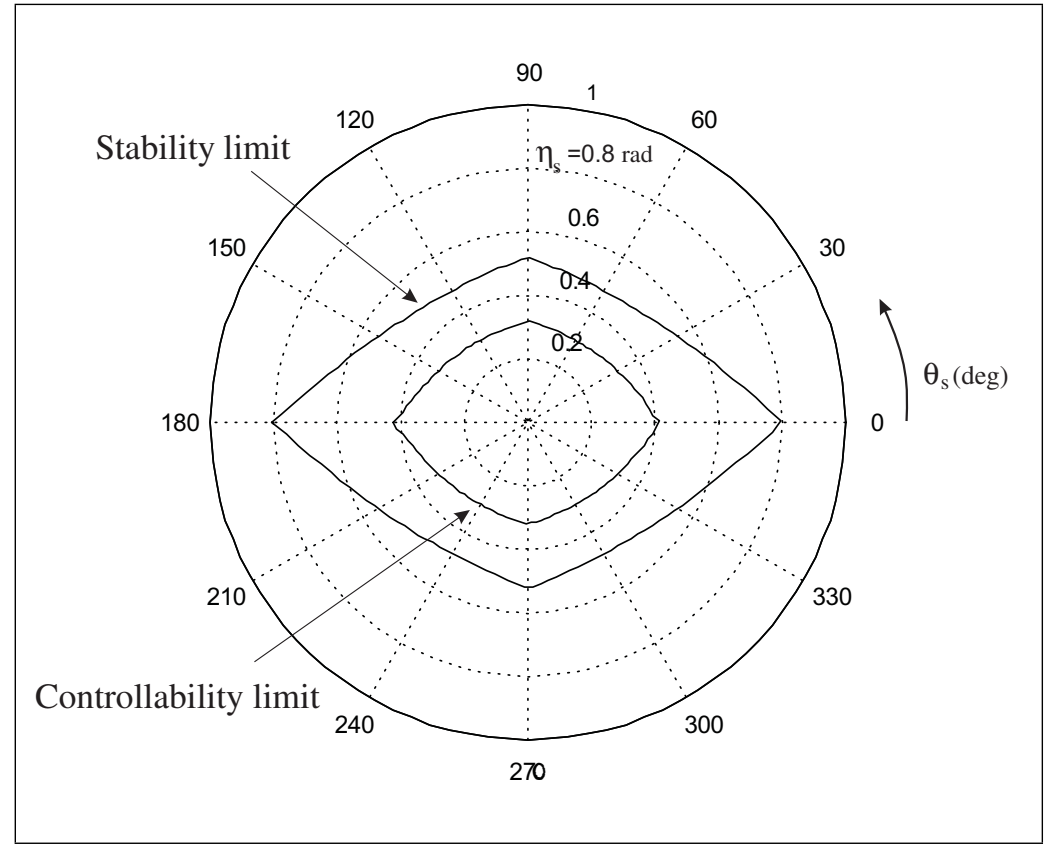

Nominal posture

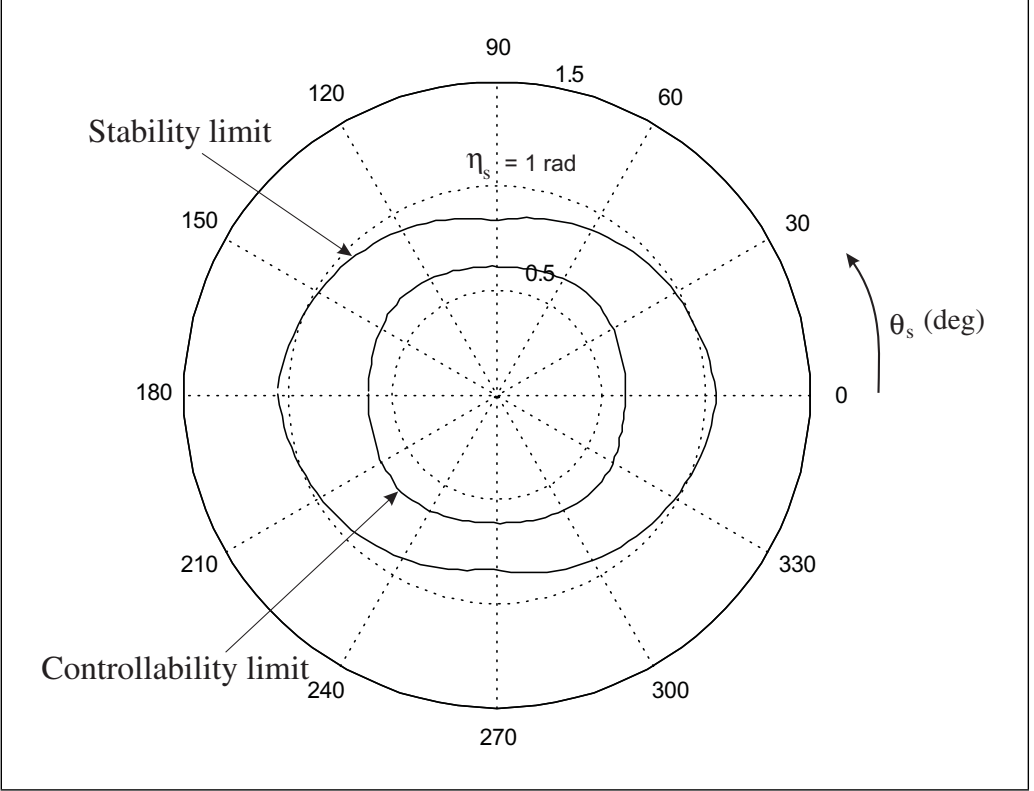

Suboptimal posture

Figure 8: Stability and controllability performance on a sloping ground 


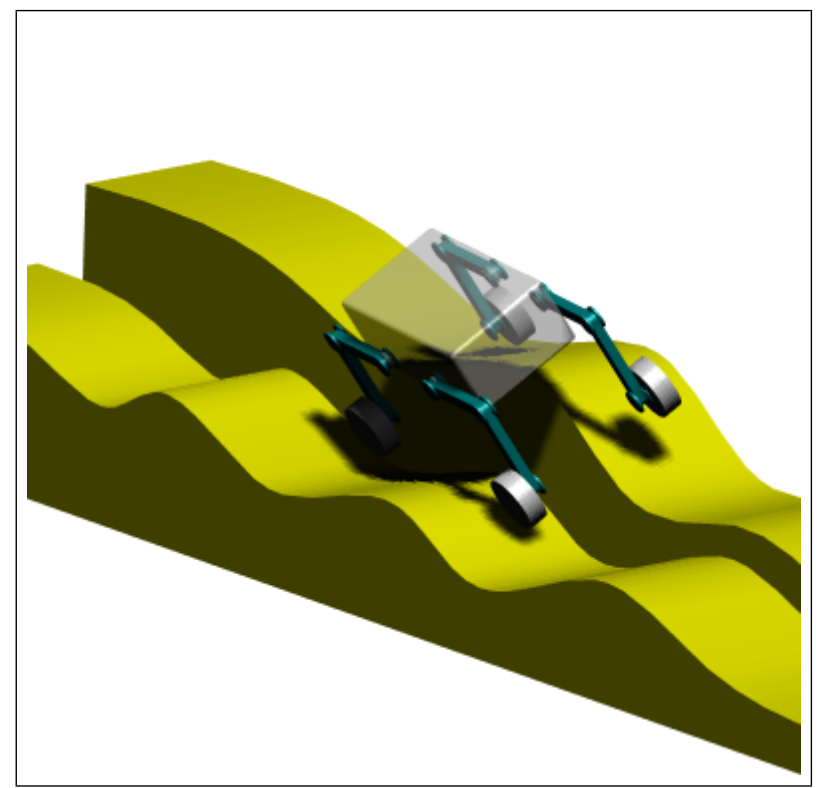

Without posture control a tipover instability occurs

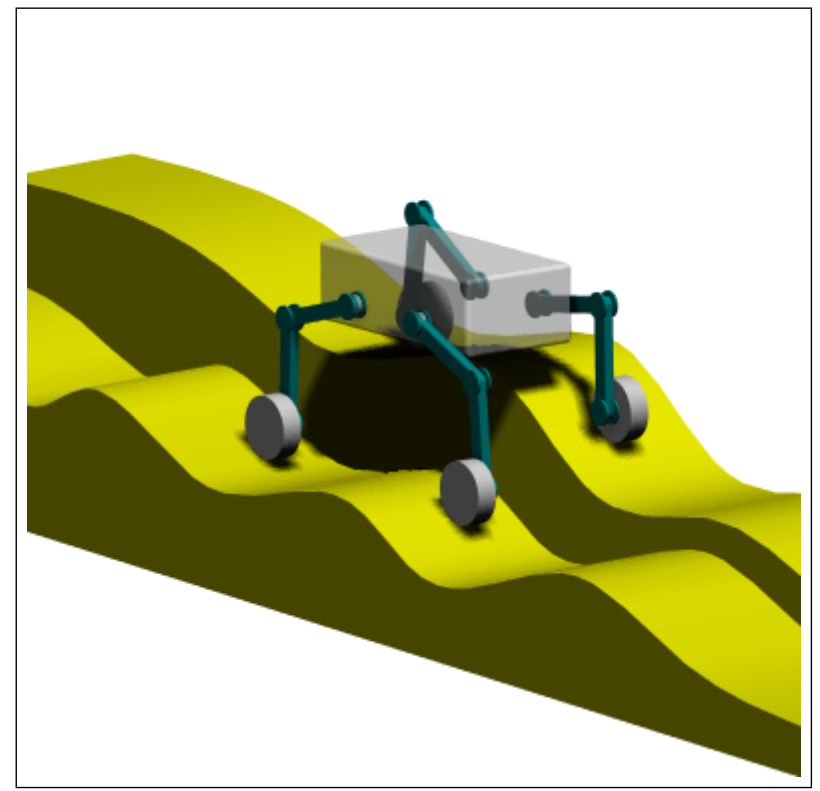

With posture control

the system stability is insured

Figure 9: Simulation of Hylos motion 


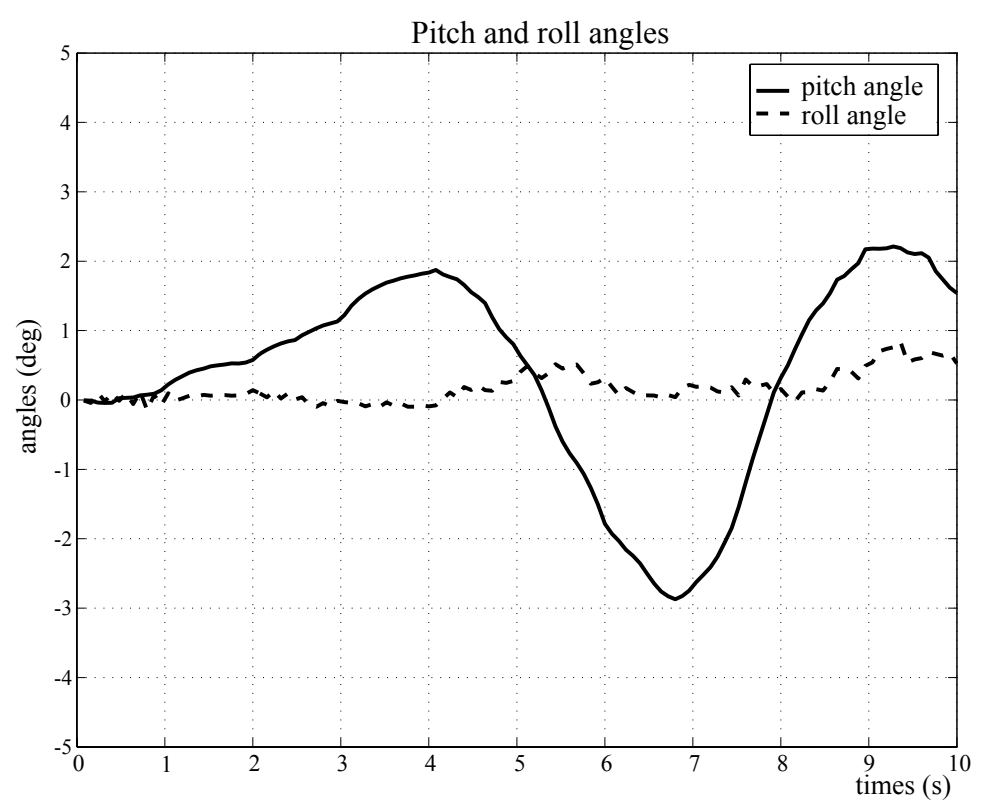

Figure 10: Pitch and roll posture control

In the Figure 11, the normal forces at each wheel-ground contact are represented, first in the case where the robot posture is not controlled (the fixed nominal posture) and secondly in the case where the robot is controlled to reach the suboptimal posture. The balance of normal forces is clearly improved in the second case. When the posture control is used the maximum standard deviation is about $16 \mathrm{~N}$ against $42 \mathrm{~N}$ without posture control, which is an enhancement of around $40 \%$ of the normal force distribution. Furthermore, we notice that without posture control all the wheels are not kept in contact with the ground, whereas with supotimal posture control the minimal contact force is higher than half the average normal force.

Last, evolution of the stability margin $m_{s}$ during robot motion is plotted in Figure 12. We recall that this corresponds to the minimum of all tipover axis angles. It is computed for both the case of suboptimal posture control and the case of fixed nominal posture. The mean stability margin of the system with posture control is $0.6 \mathrm{rad}$ whereas it is $0.15 \mathrm{rad}$ with the rigid nominal posture. The minimum stability value is $0.5 \mathrm{rad}$ using posture control and is null in the other case as tipover instability occurs. This represents a significant enhancement of the system stability.

Thus, these simulation results show the feasibility of the posture control on irregular asymmetric terrain and demonstrate a significant gain in locomotion performance. 

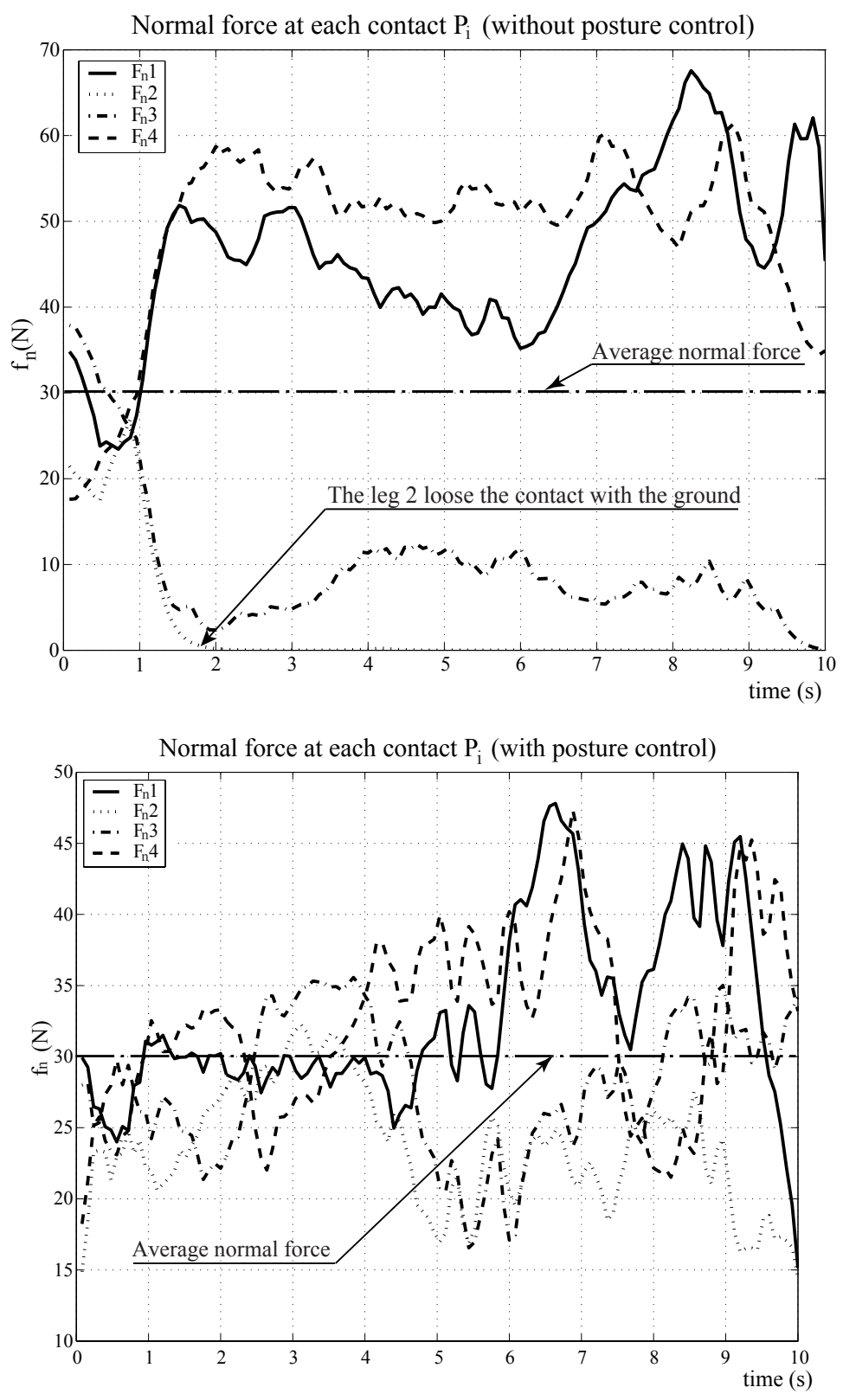

Figure 11: Forces balance criterion 


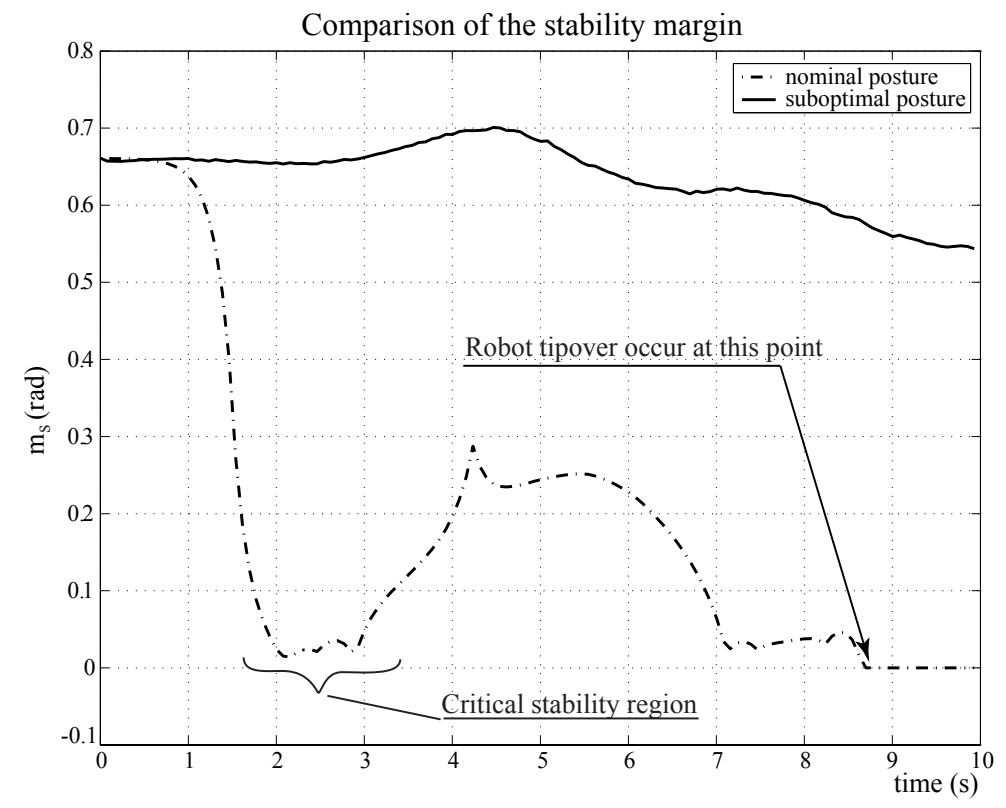

Figure 12: Stability margin criterion

\subsection{Experimental results on posture control}

The Hylos wheel-legged robot used for the experiments is approximately $60 \mathrm{~cm}$ long and weights $12 \mathrm{~kg}$. It has four legs each combining a two degrees-of-freedom suspension mechanism with a steering and driven wheel. Each leg is made of two $20 \mathrm{~cm}$ long links driven by two electrical linear actuators, and the wheel radius is $5 \mathrm{~cm}$. This mechanism can be seen as a large displacement active suspension. Hylos is equipped with a two axis inclinometer measuring the platform pitch and roll angles. One Motorola MPC555 micro-controller is dedicated to the low-level control of the 16 actuators and a PC104 board running RTLinux is used for the high-level posture control. Communication between the PC and the micro-controller is achieved through a CAN bus.

The results presented in this section consider two experimental setups. The first is composed of two successive slopes and corresponds to a nominal sloping terrain. The second is a more challenging one composed of two different terrain profiles in the left and right sagittal plane. This is an asymmetric terrain that involves a decoupled variation of the vehicle pitch and roll angles during its motion.

Experiments on slopes: In the first experiment, the robot moves straightforward at a speed of $0.15 \mathrm{~m} / \mathrm{s}$ with a constant heading angle $\theta=38^{\circ}$ on two successive slopes (see Figure 13). Thus, both the pitch and roll angles must be controlled during the motion. The two main slope angles are $18^{\circ}$ and $34^{\circ}$. The suboptimal posture is specified to be a null pitch and roll angles, with a constant ground clearance $z_{g}$ and the wheelbases computed from the method presented in Section 4.4. 


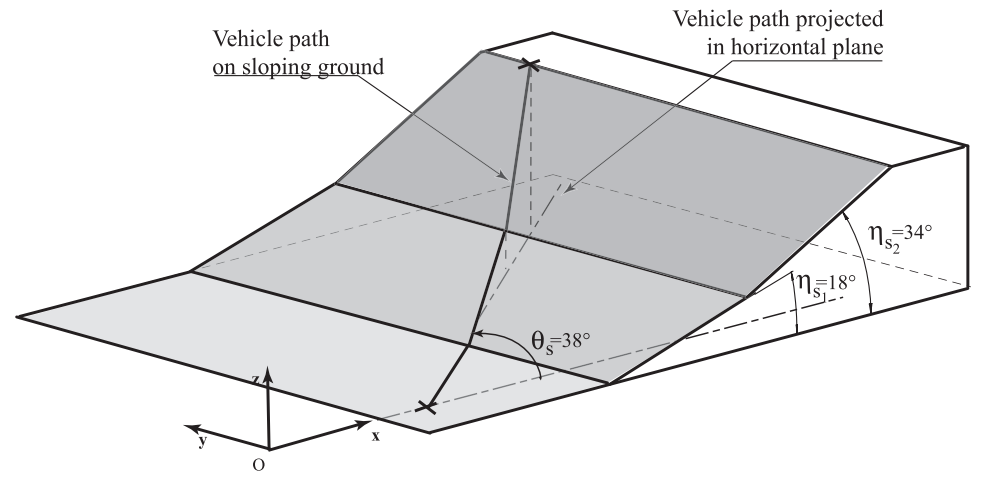

Figure 13: First experimental setup - Terrain profile
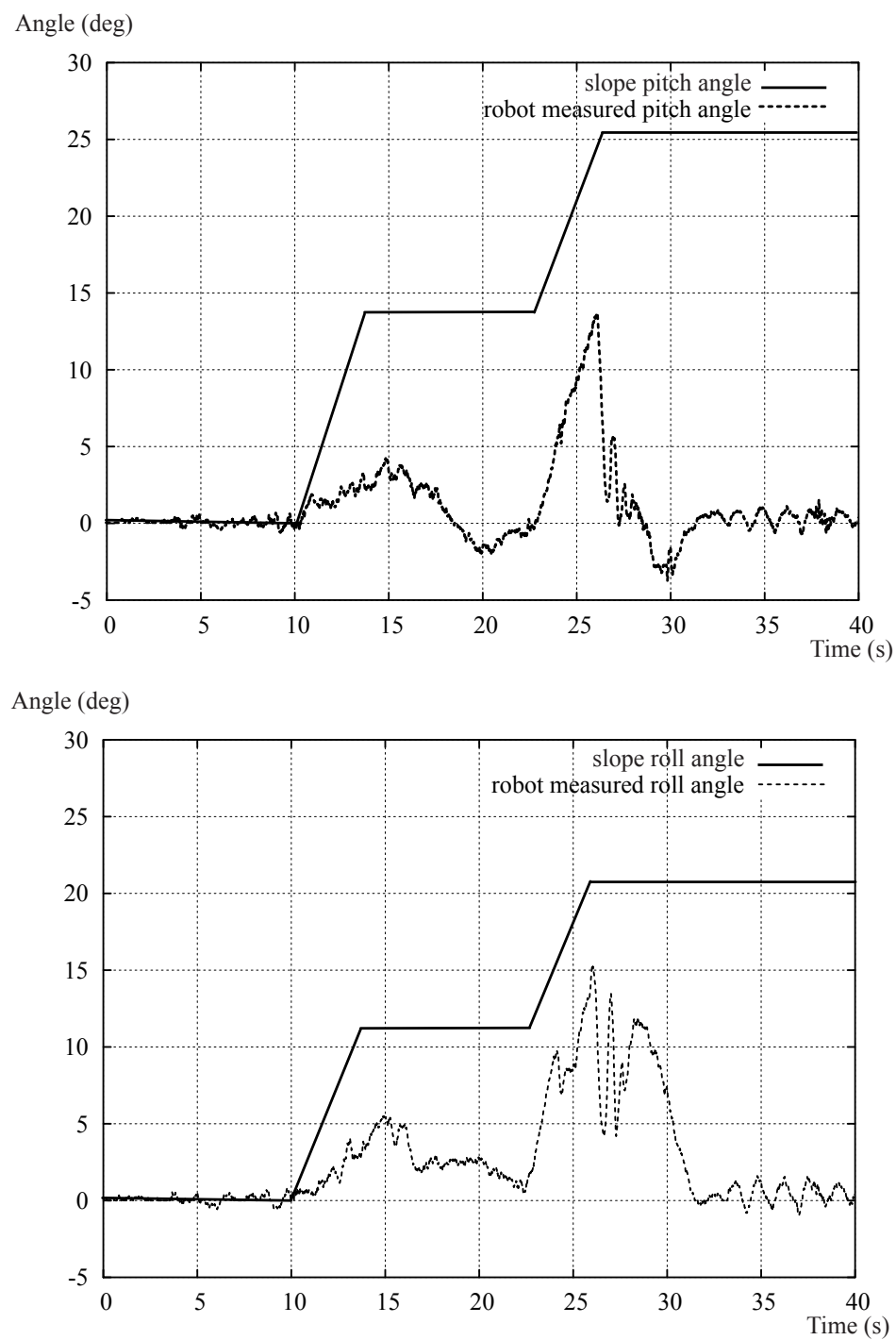

Figure 14: Experimental results of the posture control on slopes 
In Figure 14, the dashed curves represent the vehicle pitch and roll angles when posture control is active, and the solid curves is an estimate of the equivalent ground slope angles in pitch and roll directions. These angles correspond to the robot pitch and roll when it is moving with a fixed nominal posture and are computed with the Equation (29) defined in Section 5.1. The maximum error of the corrected angles (the peak on each plot) is partially due to the response time of the feedback control $(10 \mathrm{~Hz})$ and partially due to the velocity limit of the leg's actuators.

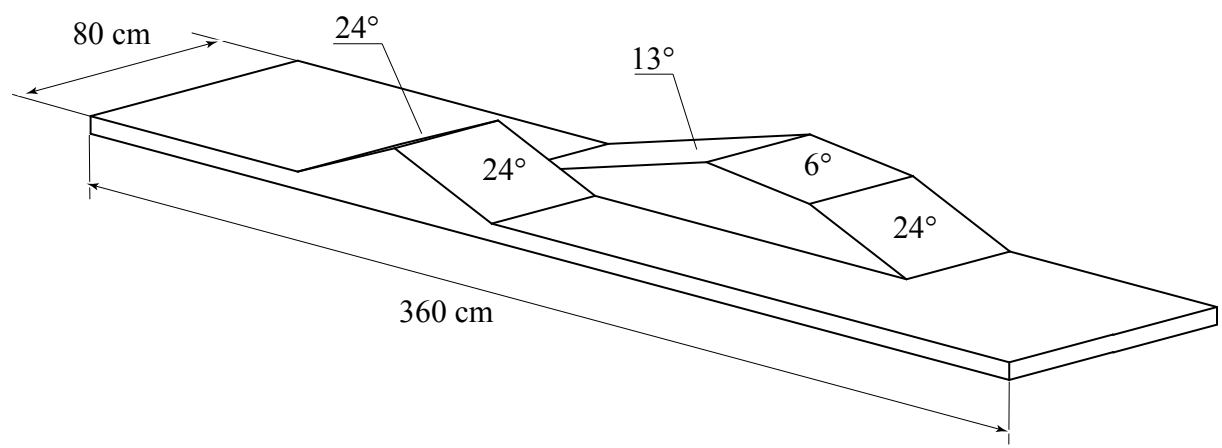

(a) Terrain profil

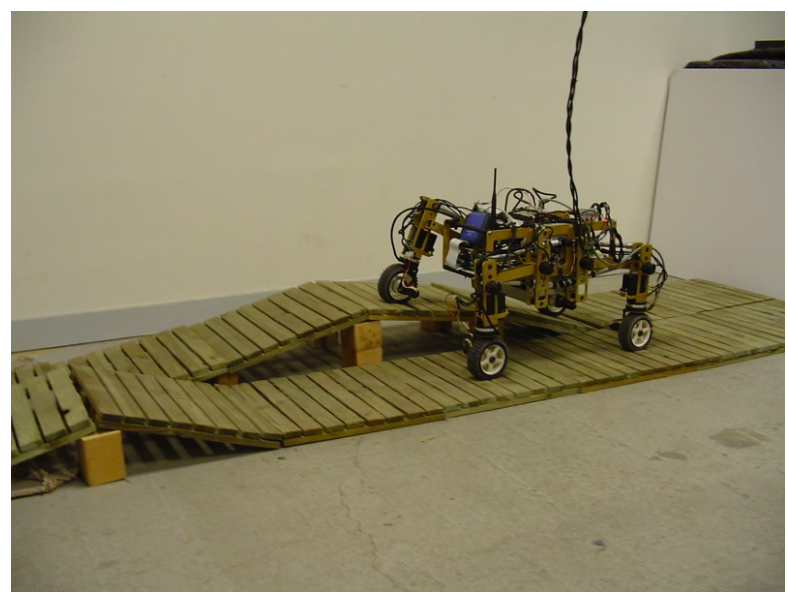

(b) Hylos posture during motion

Figure 15: Second experimental setup

Experiments on irregular terrain: In the second experiment, the robot moves straightforward at a speed of $0.08 \mathrm{~m} / \mathrm{s}$ with heading angle $\theta=0^{\circ}$ on an asymmetric irregular terrain (Figure 15). The measured pitch and roll angles are plotted in Figure 16. We compare the measured angles in the rigid nominal posture to those when the system is actively reconfigured around the suboptimal posture. These experiments show the ability of the control algorithm to maintain a certain posture. The maximum deviation of pitch and roll errors are respectively $3^{\circ}$ and $4^{\circ}$ with posture control against $10^{\circ}$ and $30^{\circ}$ without posture control (rigid nominal posture). 

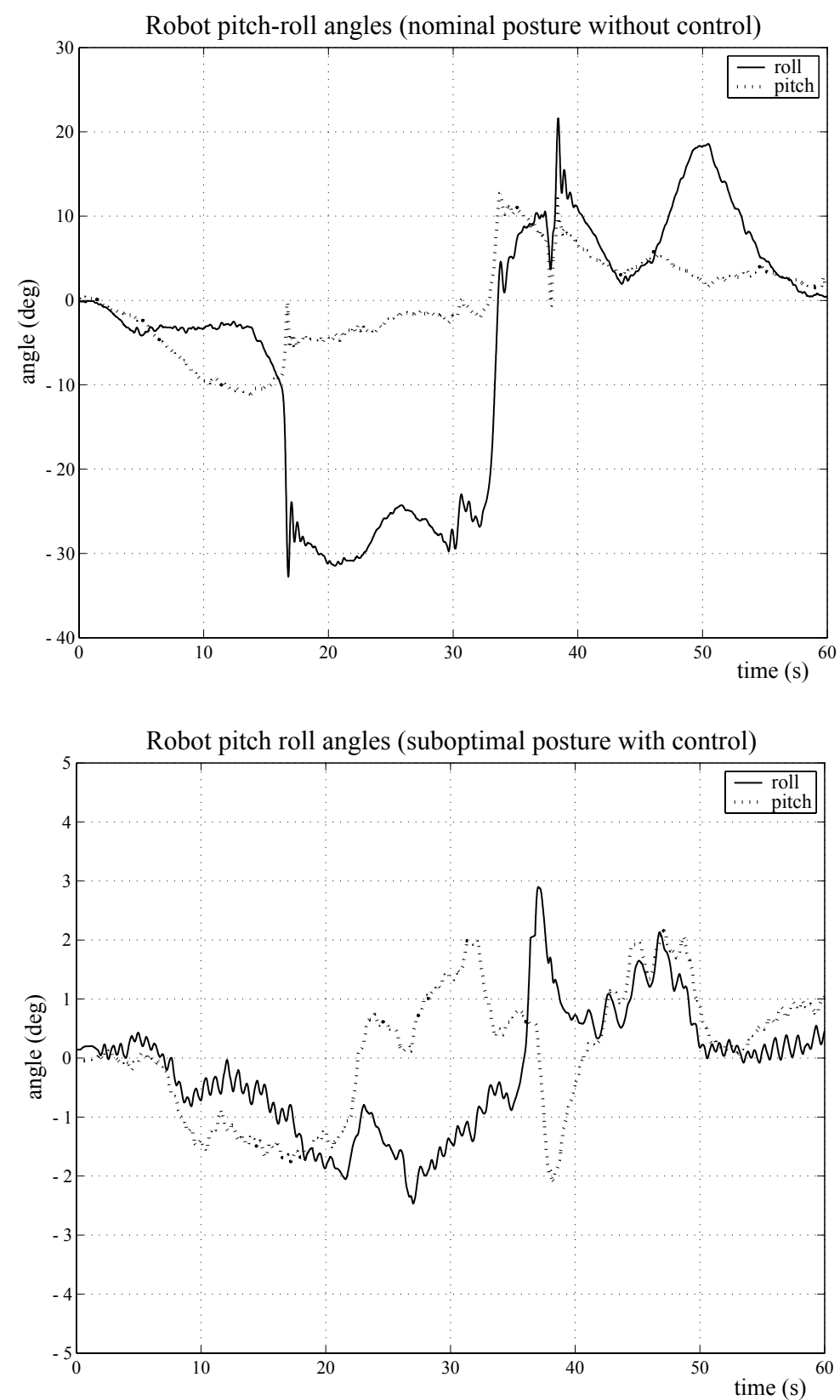

Figure 16: Experimental results of the posture control on irregular terrain 


\section{Conclusion}

In this paper, we have addressed posture control of hybrid wheel-legged vehicles. A suboptimal solution that improves both the global traction and stability performance was presented for the Hylos robot traveling on slopes.

Next, an original velocity-based posture control algorithm for a wheel-legged robot has been presented. This method is simple to implement as it needs only few sensors (a two axis inclinometer for the pitch-roll measurements and position sensors for the leg mechanism). This algorithm has been validated through simulations showing the capabilities of a vehicle to enhance its own stability in challenging environments. The practical feasibility of this control algorithm was evaluated and validated through experiments with the Hylos robot.

The next step in our work will be to study the force measurement at each wheelground contact point. These measurements will be useful for both on-line posture optimization and posture control since knowledge of the wheel-ground contact forces is a possible way to estimate the ground contact angles required for the posture optimization process. Further work will also deal with the dynamic stability control based on inertial measures for high obstacle clearance.

\section{Appendix A: Decoupled kinematics}

The rotation between platform frame and ground the frame is defined by the conventional $\operatorname{yaw}(\theta)-\operatorname{pitch}(\psi)$-roll $(\phi)$ angles and is expressed by the following rotation matrix

$$
\mathbf{R}=\left(\begin{array}{ccc}
C_{\theta} C_{\psi} & S_{\theta} C_{\psi} & -S_{\psi} \\
-S_{\theta} C_{\psi}+C_{\theta} S_{\psi} S \varphi & C_{\theta} C_{\varphi}+S_{\theta} S_{\psi} S \varphi & C_{\psi} S_{\varphi} \\
S_{\theta} S_{\varphi}+C_{\theta} S_{\psi} C \varphi & -C_{\theta} S_{\varphi}+S_{\theta} S_{\psi} C \varphi & C_{\psi} C_{\varphi}
\end{array}\right)
$$

In this case, the relation between platform rotation components $\boldsymbol{\omega}$ and rotation parameters are:

$$
\left\{\begin{array}{l}
\omega_{x}=\dot{\varphi}-\dot{\theta} \sin \psi \\
\omega_{y}=\dot{\psi} \cos \varphi+\dot{\theta} \cos \psi \sin \varphi \\
\omega_{z}=\dot{\theta} \cos \psi \cos \varphi-\dot{\psi} \sin \varphi
\end{array}\right.
$$

Then, we introduce the coupling matrix $\mathbf{D}$ such as $\mathbf{v}_{\mathbf{p}}=\mathbf{D}\left(v_{x}, v_{y}, v_{z}, \dot{\varphi}, \dot{\psi}, \dot{\theta}\right)^{\mathrm{t}}$ :

$$
\mathbf{D}=\left(\begin{array}{cccc}
\mathbf{I}_{\mathbf{3} \times \mathbf{3}} & & \mathbf{0} & \\
& 1 & 0 & -S_{\psi} \\
\mathbf{0} & 0 & C_{\varphi} & C_{\psi} S_{\varphi} \\
& 0 & -S_{\varphi} & C_{\psi} C_{\varphi}
\end{array}\right)
$$




\section{Appendix B: Hylos specific kinematics}

Considering Hylos kinematics, the position of each contact point in platform frame is approximated by:

$$
\left\{\begin{array}{l}
x_{i}= \pm l_{x}+l_{1} \cos \alpha_{i}+l_{2} \cos \left(\alpha_{i}+\beta_{i}\right) \\
y_{i}= \pm l_{y} \\
z_{i}=-\left(l_{1} \sin \alpha_{i}+l_{2} \sin \left(\alpha_{i}+\beta_{i}\right)+r_{w}\right)
\end{array}\right.
$$

where $l_{1}$ and $l_{2}$ are the length of the leg links, $l_{x}$ and $l_{y}$ are the half length and width of the platform (the sign of $l_{x}$ and $l_{y}$ depends on the position of each wheel-leg in the platform frame).

As the motion of the leg is constrained in the vehicle sagittal plane, the vector of the leg reconfiguring velocity is:

$$
\dot{\alpha}_{i} \mathbf{y} \times \mathbf{a}_{\mathbf{i}}+\dot{\beta}_{i} \mathbf{y} \times \mathbf{b}_{\mathbf{i}}=\left(\dot{x}_{i}, 0, \dot{z}_{i}\right)^{\mathrm{t}}
$$

and the time derivative of $x_{i}$ and $z_{i}$ :

$$
\left\{\begin{array}{l}
\dot{x}_{i}=-\left(l_{1} \sin \alpha_{i}+l_{2}\left(\sin \alpha_{i}+\beta_{i}\right)\right) \dot{\alpha}_{i}-l_{2} \sin \left(\alpha_{i}+\beta_{i}\right) \dot{\beta}_{i} \\
\dot{z}_{i}=-\left(l_{1} \cos \alpha_{i}+l_{2}\left(\sin \alpha_{i}+\beta_{i}\right)\right) \dot{\alpha}_{i}-l_{2} \cos \left(\alpha_{i}+\beta_{i}\right) \dot{\beta}_{i}
\end{array}\right.
$$

Then, the velocity terms $\mathbf{v}_{\mathbf{i}}^{\prime}$ used in Equation (23) is:

$$
\left\{\begin{array}{l}
v_{i_{x}}^{\prime}=v_{x}+\omega_{y} z_{i}-\omega_{z} y_{i}+\dot{x}_{i} \\
v_{i_{y}}^{\prime}=v_{y}+\omega_{z} x_{i}-\omega_{x} z_{i} \\
v_{i_{z}}^{\prime}=v_{z}+\omega_{x} y_{i}-\omega_{y} x_{i}+\dot{z}_{i}
\end{array}\right.
$$

The projection in the vehicle frame $\mathcal{R}$ of the unit vector $\boldsymbol{\sigma}_{\mathbf{i}}$ of each wheel axis gives:

$$
\boldsymbol{\sigma}_{\mathbf{i}}=\left(\begin{array}{c}
-\sin \left(\gamma_{i}\right) \sin \left(\alpha_{i}+\beta_{i}\right) \\
\cos \left(\gamma_{i}\right) \\
\sin \left(\gamma_{i}\right) \cos \left(\alpha_{i}+\beta_{i}\right)
\end{array}\right)
$$

By solving Equation (19) $\left(\boldsymbol{\sigma}_{\mathbf{i}}^{\mathrm{t}} \mathbf{v}_{\mathbf{i}}^{\prime}=0\right)$, we obtain:

$$
-\sin \left(\gamma_{i}\right)\left(v_{i_{x}}^{\prime} \sin \left(\alpha_{i}+\beta_{i}\right)-v_{i_{z}}^{\prime} \cos \left(\alpha_{i}+\beta_{i}\right)\right)+\cos \left(\gamma_{i}\right) v_{i_{y}}^{\prime}=0
$$

which gives the steering angle value $\gamma_{i}$ of Equation (23).

In the time derivative of posture vector $\dot{\mathbf{p}}$, the $\dot{z}_{g}$ component is a function of $\mathbf{v}_{\mathbf{p}}$. We remind that $z_{g}$ is defined as the average of the contacts height $z_{i}$ (see Section 4.1). Thus, $\dot{z}_{g}$ is computed as follows:

$$
\dot{z}_{g}=\sum_{i} \frac{\dot{z}_{i}}{4}
$$


where $z_{i}=\mathbf{r}_{\mathbf{i}}{ }^{\mathrm{t}} \mathbf{z}$.

The time derivative of $z_{i}$ is:

$$
\dot{z}_{i}=\dot{\mathbf{r}}_{\mathbf{i}}^{\mathrm{t}} \mathbf{z}+\mathbf{r}_{\mathbf{i}}^{\mathrm{t}} \dot{\mathbf{z}}
$$

where $\dot{\mathbf{r}}_{\mathbf{i}}$ is the velocity of the contact point relative to the vehicle frame.

So, by considering the pure rolling condition introduced in Section 3 (non-slipping and contact constraints), this leads to:

$$
\dot{\mathbf{r}}_{\mathbf{i}}=\mathbf{v}_{\left(\mathrm{P}_{\mathrm{i}} / \mathcal{R}_{0}\right)}-\mathbf{v}_{\left(\mathrm{G} / \mathcal{R}_{0}\right)}=-\mathbf{v}
$$

Since the time derivative of $\mathbf{z}$ vehicle frame vector is $\dot{\mathbf{z}}=\mathbf{S}(\boldsymbol{\omega}) \mathbf{z}$, we obtain:

$$
\dot{z}_{i}=-\mathbf{v}^{\mathrm{t}} \mathbf{z}+\mathbf{r}_{\mathbf{i}}^{\mathrm{t}}(\mathbf{S}(\boldsymbol{\omega}) \mathbf{z})
$$

And finally, the time derivative of $z_{g}$ is computed as follows:

$$
\dot{z}_{g}=-\mathbf{v}^{\mathrm{t}} \mathbf{z}+\frac{1}{4} \sum_{i} \mathbf{r}_{\mathbf{i}}{ }^{\mathrm{t}}(\mathbf{S}(\boldsymbol{\omega}) \mathbf{z})
$$

\section{References}

[1] G. Andrade, F. BenAmar, Ph. Bidaud, and R. Chatila. Modeling wheel-sand interaction for optimization of a rolling-peristaltic motion of a marsokhod robot. In International Conference on Intelligent Robots and Systems, pages 576-581, 1998.

[2] J. Balaram. Kinematic state estimation for a mars rover. Robotica, 18(3):251-262, 2000 .

[3] F. BenAmar, V. Budanov, Ph. Bidaud, F. Plumet, and G. Andade. A high mobility redundantly actuated mini-rover for self adaptation to terrain characteristics. In $3 r d$ International Conference on Climbing and Walking Robots, pages 105-112, Madrid, Spain, 2000.

[4] J.J. Craig. Introduction to Robotics. Addison-Wesley, 1989.

[5] T. Estier, Y. Crausaz, B. Merminod, M. Lauria, R. Piguet, and R. Siegwart. An innovative space rover with extended climbing abilities. In International Conference on Robotics in Challenging Environments, Albuquerque, USA, 2000.

[6] A. Ghasempoor and N. Sepehri. A measures of machine stability for moving base manipulators. In IEEE Int. Conference on Robotics and Automation, pages 22492254, 1995. 
[7] Ch. Grand, F. BenAmar, P. Bidaud, and G. Andrade. A simulation system for behaviour evaluation of off-road mobile robots. In 4 th International Conference on Climbing and Walking Robots, pages 307-314, Germany, 2001.

[8] A. Halme, I. Leppänen, S. Salmi, and S. Ylönen. Hybrid locomotion of a wheellegged machine. In International Conference on Climbing and Walking Robots, Madrid, Spain, 2000.

[9] S. Hirose and H. Takeuchi. Study on roller-walk (basic characteristics and its control). In IEEE Int. Conference on Robotics and Automation, pages 3265-3270, 1996.

[10] S. Hirose, H. Tsukagoshi, and K. Yoneda. Normalized energy stability margin and its contour of walking vehicles on rough terrain. In IEEE Int. Conference on Robotics and Automation, pages 181-186, 2001.

[11] K. Iagnemma and S. Dubowsky. Vehicle wheel-ground contact angle estimation: with application to mobile robot traction control. In Proceedings of the 7th Int. Symposium on Advances in Robot Kinematics, pages 137-146, 2000.

[12] K. Iagnemma, A. Rzepniewski, S. Dubowsky, and P. Schenker. Control of robotic vehicles with actively articulated suspensions in rough terrain. Autonomous Robots, 14(1):5-16, 2003.

[13] A.L. Kemurdjian. Planet rover as an object of the engineering design work. In IEEE International Conference on Robotics and Automation, pages 140-145, Belgium, 1998.

[14] V.R. Kumar and K.J. Waldron. Force distribution in closed kinematic chains. IEEE Journal of Robotics and Automation, 4(6):657-663, 1988.

[15] R. McGhee and A. Frank. On the stability properties of quadruped creeping gait. Mathematical Bioscience, 3:331-351, 1968.

[16] R.B. McGhee. Finite state control of quadruped locomotion. In Proceedings of the International Symposium on External Control of Human Extremities, 1966.

[17] D.A. Messuri and C.A. Klein. Automatic body regulation for maintaining stability of a legged vehicle during rough terrain locomotion. IEEE Journal of Robotics and Automation, RA-1(3):132-141, 1985.

[18] F. Michaud and al. Azimut, a leg-track-wheel robot. In IEEE Int. Conference on Intelligent Robots and Systems, pages 2553-2558, 2003.

[19] E.G. Papadopoulos and D.A. Rey. A new mesure of tipover stability for mobile manipulators. In IEEE Int. Conf. on Robotics and Automation, pages 3111-3116, 1996. 
[20] E. Rollins, J. Luntz, A. Foessel, B. Shamah, and W. Whittaker. Nomad: a demonstration of the transforming chassis. In IEEE International Conference on Robotics and Automation, pages 611-617, Belgium, 1998.

[21] U. Saranli, M. Buehler, and D.E. Koditschek. Rhex: a simple and highly mobile hexapod robot. Int. J. Robotics Research, 20:616-631, 2001.

[22] S.M. Song and K.J. Waldron. Machines that walk: the adaptative suspension vehicle. The MIT press, 1989.

[23] S.V. Sreenivasan and K.J. Waldron. Displacement analysis of an actively articulated wheeled vehicule configuration with extensions to motion planning on uneven terrain. Transactions of the ASME, 118(6):312-317, 1996.

[24] S.V. Sreenivasan and B.H. Wilcox. Stability and traction control of an actively actued micro-rover. Journal of Robotics Systems, 11(6):487-502, 1994.

[25] R. Volpe. Rocky 7: A next generation mars rover prototype. Journal of Advanced Robotics, 11(4):341-358, 1997. 